\title{
Evaluación de tres momentos de riego en el cultivo de la sábila en el Municipio Colina, Falcón. Venezuela
}

\section{Evaluation of three moments of irrigation farming in the aloe of Colina Municipality, Falcon. Venezuela}

\author{
Víctor Alfonso Blanco Bello \\ vicolines@hotmail.com \\ Universidad Politécnica Territorial de Falcón Alonso Gamero \\ Venezuela \\ Juan Pedro Machado Castillo \\ imachadoc@udg.co.cu \\ Universidad de Granma \\ Cuba \\ Norge Tornes Olivera \\ ntorneso@udg.co.cu \\ Universidad de Granma \\ Cuba
}

Recibido: 30 de noviembre del 2016

Aprobado: 12 de febrero del 2017

\section{RESUMEN}

La investigación se realizó en la finca La Estrella, Municipio Colina, estado Falcón, con el objetivo de evaluar tres momentos de riego en el cultivo de la sábila (Aloe vera L.). Se utilizó un diseño en franjas con 4 tratamientos y 4 repeticiones: $T_{0}$ (sin riego), $T_{1}$ (riego $8 \mathrm{am}$ ), $\mathrm{T}_{2}$ (riego $3 \mathrm{pm}$ ) y $\mathrm{T}_{3}$ (riego $8 \mathrm{pm}$ ). Se evaluaron las variables largo, grosor, ancho, peso de la hoja, peso del gel y se estimó el rendimiento en hoja y gel. La aplicación de riego en el cultivo de la sábila favorece el crecimiento y desarrollo de la planta. Con el riego nocturno $(8.00 \mathrm{pm})$, se alcanzan los mejores resultados de los indicadores del crecimiento de la planta, así como de peso de la hoja $(514,62 \mathrm{~g})$ y de gel $(326,49 \mathrm{~g})$ con un rendimiento estimado en hoja y gel de $61,752 \mathrm{Mg}^{-h^{-1}}{ }^{-1}$ corte $^{-1}$ año

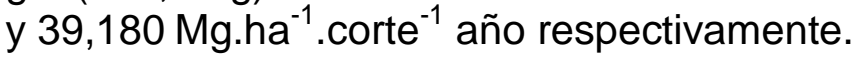

Descriptores: Sábila; riego por micro-aspersión; producción de gel; hoja. 


\section{ABSTRACT}

The research was carried in farm La Estrella, Colina Municipality, Falcon state, with the objective of evaluating three irrigation moments in the crop of Aloe vera L. A striped design was used with 4 treatments and 4 replicates: T0 (without irrigation), T1 (irrigation $8 \mathrm{am}$ ), T2 (irrigation $3 \mathrm{pm}$ ) and T3 (irrigation $8 \mathrm{pm}$ ). The variables length, thickness, width, leaf weight, gel weight and leaf and gel yield were evaluated. The application of irrigation in the cultivation of aloe vera favors the growth and development of the plant. With night watering $(8.00 \mathrm{pm})$, the best results of the plant growth indicators, as well as leaf weight $(514.62 \mathrm{~g})$ and gel $(326.49 \mathrm{~g})$ are achieved with an estimated yield in leaf and gel of $61,752 \mathrm{Mg} \cdot$ ha-1. cut $^{-1}$ year and 39,180 Mg.ha-1. cut $^{-1}$ year respectively

Descriptors: Aloe vera: micro-spray irrigation: production gel: sheet.

\section{INTRODUCCIÓN}

El cultivo de sábila (Aloe vera L.) es un cultivo con una vasta tradición en Falcón (Venezuela), sin embargo aún se continúa explotando de manera artesanal en plantaciones de pequeña escala y siguiendo un procesamiento agroindustrial rudimentario, donde se extrae fundamentalmente la pasta como principal producto comercializable, a la cual corresponde el acíbar (exudado de la hoja) deshidratado mediante técnicas de cocción hasta alcanzar un sólido de color negruzco con $4 \%$ de humedad (Piña et.al., 2005).

La pasta tuvo un importante auge entre la década de los 70 y 80, cuando Falcón llegó a exportar hasta13\% del mercado mundial (Bisceglia, 1999). Sin embargo en la actualidad ha sido desplazado por importantes plantaciones tecnificadas que se han establecido en Costa Rica, República Dominicana y México, donde se utilizan modernas técnicas de cultivo y se han orientado esas plantaciones hacia la obtención de gel (Piña, 2005).

El estado Falcón es el mayor productor del cultivo de sábila (Aloe vera L.) para la producción de acíbar en el país (Piña et al., 2005), por presentar clima semiárido en gran parte de su extensión, sin embargo estas condiciones dificultan la producción del cultivo de sábila para gel, ya que aunque la planta tolera el estrés hídrico, para este tipo de producción necesita condiciones adecuadas de humedad para alcanzar un máximo 
desarrollo. En relación a lo anterior, González; Puertas; Jiménez; Danger y López (2018) señalan que:

La zábila Aloe vera L. es una planta suculenta de múltiples aplicaciones terapéuticas y de importancia socioeconómica para las regiones áridas y semiáridas de Venezuela, esta especie cuenta con características articulares que hacen que sea un cultivo productivo en condiciones donde prevalecen la baja fertilidad y escasez de agua, de allí que surgen nuevas propuestas para el logro sustentable y eficiente de dicho rubro en el estado Falcón ( $p, 214)$.

Por estas razones en el territorio la producción de gel está solo limitada a la época de lluvia, donde se realiza una sola cosecha con rendimientos anuales bajos (Piña, 2005). La utilización del riego a las plantaciones sería una solución a esta problemática, pero hasta el momento no se conocen técnicas y parámetros de riegos adecuados para la producción óptima de gel en Venezuela.

El objetivo del trabajo es evaluar tres momentos de riego en el cultivo de la sábila (Aloe vera L.) en el sector Las Ventosas municipio Colina, Falcón Venezuela.

\section{MATERIALES Y MÉTODOS}

\section{Localización del experimento}

La unidad de producción donde se realizó la investigación está ubicada geográficamente $11^{\circ} 26^{\prime \prime} 03^{\prime}$ latitud y $69^{\circ} 31^{\prime \prime} 54^{\prime}$ longitud y $78 \mathrm{msnm}$, en el sector de Las Ventosas parroquia La Vela de Coro, municipio Colina del estado Falcón a 4 kilómetros de la vía nacional Morón- Coro; dicha unidad posee el nombre de "La Estrella" propiedad del señor Serafín Ayala. La unidad de producción cuenta con una superficie total 17,5 hectáreas y 5 se encuentra cultivada de sábila con una densidad de 20,000 plantas/ha.

\section{Características Edafo-climáticas}

Relieve: El municipio corresponde a la Entidad Natural Anticlinario y Surco Central de Falcón (MAC; 1975), y cuenta con tres tipos de relieves: escabrosos y accidentados 
ondulados y planos. Presentado la zona de estudio topografía plana con pendiente menor al $2 \%$, además de micro relieves y áreas onduladas poco pronunciadas con suelos.

Suelos: arcillo limosos, con una profundidad promedio 60 centímetros, drenaje externo moderado, el interno y la permeabilidad es lenta. $\mathrm{El} \mathrm{pH}$ es neutro a ligeramente alcalino. (MAC; 1975).

Recurso hídrico: La unidad de producción posee un tanque con capacidad de $54 \mathrm{~m}^{3}$ el cual se surte del agua proveniente del suministro de agua por tubería del sistema público.

Clima: presenta clima semiárido con temperatura: entre $27{ }^{\circ} \mathrm{C}$ y $28 \stackrel{\circ}{\circ}$, siendo los meses más cálidos agosto, septiembre, y octubre, menos cálido enero, febrero y marzo (MAC; 1975).

Precipitaciones: la pluviométrica es de carácter bimodal, donde las máximas precipitaciones se presentan en los meses de octubre y noviembre con valores de 105,18 y 59,65 mm respectivamente, y las mínimas en los meses enero y abril con valores de 24,44 y 30,29 mm respectivamente. (MAC; 1975).

\section{Registros climatológicos del experimento.}

Los registros de precipitaciones se tomaron de un pluviómetro artesanal instalado en el campo según las normas de la Organización mundial de meteorología (OMM), (Monasterio, et al., 2008). Las temperaturas se tomaron de lecturas directas de un termómetro marca "Nahita".

\section{Diseño experimental.}

El experimento se realizó con un diseño en franja, donde está establecido el cultivo de sábila desde hace 3 años presentando características de estrés hídrico severo (hojas delgadas y rojizas y con porte bajo en general). Para la preparación del área experimental se desarrollaron las labores agronómicas de limpieza y deshije de las 
plantas, desinfección incorporando cal agrícola y Metarrizum sp en dosis de $60 \mathrm{~g} / \mathrm{ha}$ para la prevención de plagas.

La investigación contó con un área total $336 \mathrm{~m}^{2}$, con cuatro réplicas y cuatro tratamientos: $T_{0}$ (control sin riego), $T_{1}$ (riego 8:00 am), $T_{2}$ (riego 3:00 pm) y $T_{3}$ (riego 8:00 pm). El total de plantas fueron de 672, establecidas a una distancia de 0,5 metros entre planta y 1 metro entre hilera, cada tratamiento contó de 168 plantas con 42 plantas por repetición, con un área de $21 \mathrm{~m}^{2}$. El área de muestreo por tratamiento es de $84 \mathrm{~m}^{2}$. Se realizó un muestreo en cuatro plantas por repetición, seleccionadas aleatoriamente, para un total de 16 plantas; tomadas de los dos surcos centrales para evitar el efecto de borde. En dichas plantas se realizaron las mediciones de las diferentes variables morfológicas como: largo, ancho y grosor de la hoja, tomando la quinta hoja de las plantas seleccionadas para dichas mediciones; siguiendo el método sugerido por Pulido (2014), el cual consiste básicamente en contar del centro hacia fuera a partir de la última hoja emitida hasta llegar a la quinta y marcarla con cinta.

\section{Técnica de riego.}

Se aplicó a través de la técnica de riego localizado (micro aspersión) cubriendo la necesidad hídrica del cultivo con un régimen de 1 litro de agua/planta/ por día siguiendo la metodología de Pulido, (2014). Para ello se utilizo micro aspersores de marca Agrid con las siguientes características: Caudal 0,6 L/min, radio de tiro: 1,5 m, presión de trabajo de 2 bares. Con un marco de riego en disposición en cuadrado con espaciamiento entre ellos de $1,5 \mathrm{~m}$, solapamiento de $100 \%$, coeficiente de uniformidad de $90 \%$ y con la eficiencia de aplicación de $85 \%$. Aplicando una lámina de riego de 7 $\mathrm{mm}$ en un tiempo de 20 min con frecuencia de 3 días, para ello se tomaron en cuenta las características edafoclimáticas presente de la zona.

La tubería principal es de polietileno con un diámetro de $40 \mathrm{~mm}$, las tuberías secundaria de $25 \mathrm{~mm}$ en las cuales se instalaron los microaspersores, para el bombeo se utilizó 
una electro bomba con un motor modelo SIEMENS con potencia de $1 \mathrm{Hp}$ y $3520 \mathrm{rpm}$, con una carga de trabajo de $25 \mathrm{mca}$. La fuente del suministro de agua es un tanque de concreto con una capacidad de almacenamiento de $54 \mathrm{~m}^{3}$, el mismo es abastecido por tubería del embalse El Isiro.

\section{Variables morfológicas evaluadas.}

La medición de las variables (largo, ancho, grosor de la hoja) se realizó a partir de los 10 días después de la aplicación del tratamiento, efectuándose 10 mediciones en un lapso 3 meses. Se realizaron curvas relacionales de la dinámica de crecimiento para cada una de las variables en estudio. Dichas mediciones se realizaron siguiendo la metodología descrita por Añez y Vásquez, (2005):

Largo de la hoja (cm): se medió con una regla graduada desde la inserción de la hoja hasta el ápice.

Ancho de la hoja (cm): se efectuó con el uso de un vernier a $10 \mathrm{~cm}$ de la inserción al ápice.

Grosor de la hoja (cm.): se efectuó con el uso de un vernier midiendo el grosor a 10 cm de la inserción al ápice.

\section{Variable de producción.}

\section{Rendimiento en biomasa fresca de las hojas ( $\mathrm{kg} / \mathrm{ha})$.}

Se determinó después de cosechar con el uso de un cuchillo de acero inoxidable desinfectado con agua de cloro al 1 \% (López et al., 2010) tres hoja adultas (externas) por planta, para un total de 48 hojas por tratamiento, dichas hojas se identificaron y se colocaron dentro de una cesta plástica para su pesado en una balanza eléctrica. Así mismo, teniendo en cuenta la densidad de plantación del cultivo de sábila (20,000 plantas por hectárea), y considerando dos cortes anuales, se calculó el rendimiento total de hoja y gel para el ensayo, siguiendo la metodología de Añez y Vásquez (2005), así como de Figueredo y Morales (2010). 


\section{Peso de gel.}

Para determinar el peso del gel, se procedió con la ejecución de la metodología utilizada en la Fábrica para Procesamiento de Sábila de Venezuela S.A (SABILVEN S.A, 2005), la cual consiste en el lavado de las hojas cosechadas con agua para eliminar todos los residuos de tierra e impurezas, luego se fileteó de forma manual, donde se eliminaron los bordes espinosos y la epidermis de la hoja para evitar la alteración química de la misma, posteriormente se procedió al pesaje, de las muestras de cada tratamiento en la balanza electrónica.

A partir de los resultados de los pesos de Gel y de la hoja, se estimó el rendimiento teniendo en cuenta dos cortes anuales.

\section{Análisis estadístico}

Para comparar los tratamientos en cuanto a las variables estudiadas se realizaron análisis de varianzas clasificación simple y las medias se compararon mediante la prueba de Tukey $(p \leq 0,05)$. El paquete utilizado fue STATISTICA V-7.0 sobre Windows 2007.

\section{Resultados y Discusión}

Al evaluar el largo de la hoja de sábila en tres momentos de riego (Fig.1), se observa que a partir de los diez días de aplicado el riego, en todos los tratamientos incluyendo el control (sin riego) incrementaron el crecimiento, sin embargo se observa que los tratamientos con los diferentes momentos de riego sus incrementos fueron superior al control con diferencias significativas con este. El efecto de los tratamientos se evidencia claramente al pasar los 80 días de la aplicación de los tratamientos, donde el efecto del riego nocturno (T3) es significativo sobre el aumento del largo de la hoja en comparación con el resto de los tratamientos. 
Este comportamiento guarda relación con el metabolismo de la planta (ácido de las crasuláceas, CAM) que permite la conservación del agua dentro del tejido, y por lo tanto, la resistencia al estrés hídrico (Rodríguez et al., 2007), lo que justifica su continuo crecimiento aún en condiciones bajas de humedad del suelo, como ha sucedido en esta experiencia, donde ocurrieron escasas precipitaciones, sin embargo con el transcurso de los días los tratamientos donde se realizaron aplicaciones del riego, se incrementó el crecimiento, lo que pudiera traducirse en la obtención de un gel de buenas condiciones. Al respecto Geydan y Melgarejo (2005) señalan que esta planta por la noche abre sus estomas para adquirir el dióxido de carbono y la humedad del ambiente y por el día cierra sus estomas para evitar la pérdida de agua, en ellas no se realiza a la vez como en la mayoría de las plantas, sino que en el proceso llevado a cabo por la noche capta CO2 y acumula ácido málico en las vacuolas. Por el día el ácido málico sale de la vacuola y libera dióxido de carbono internamente y así realizan la fotosíntesis. Este tipo de plantas pierde, tan solo, entre 50-100 g de agua frente al resto de las plantas que puede perder entre 200 a 500 gramos.

Con relación al ancho de la hoja (Fig. 2), también se puede observar que el tratamiento 3 fue el que presentó mejor comportamiento, seguido por el tratamiento 1 y 2 , sobre todo después de los 80 días del inicio de la aplicación de riego, donde todos los tratamientos bajo riego superaron al control.

Estos resultados indican que el suministro adecuado de agua en horas nocturnas favorece el crecimiento de la planta, ya que en la sábila por su metabolismo, por la noche capta $\mathrm{CO} 2$ y acumula ácido málico en las vacuolas, lo que favorece el proceso fotosintético y el posterior procesamiento de formación de gel para la fabricación de productos aptos para la industria farmacéutica, cosmética y alimentaria (Bozzi et al., 2007).

En la figura 3 se ilustra el grosor de la hoja de sábila en tres momento de riego, se observar que a partir de los 50 días de iniciado el riego, se produce las diferencias en el 
grosor entre los riegos nocturno y el de las 8.00 am con el riego de las $3.00 \mathrm{pm}$ y el tratamiento control (sin riego).

Con este resultado, se evidencia que después de los 50 días de la aplicación de los tratamientos, hay una respuesta mayor de la planta de sábila a la aplicación de los diferentes momentos de aplicación de agua, siendo mayor la respuesta en el tratamiento T3 (riego 8 pm) y las primeras horas de la mañana T1 (riego 8 am) que es cuando la planta es menos afectada por las altas temperaturas y radiaciones solares.

En la tabla 1 se muestra el análisis estadístico realizado a los indicadores del crecimiento y rendimiento en los diferentes momentos de riego, donde se observa que en el largo de la hoja se alcanza el mayor valor en el riego a las 8.00 am, con diferencias significativas con el control, no así con el resto de los tratamientos con riego, los cuales superan también significativamente al control, con la excepción del riego a las $3.00 \mathrm{pm}$.

Tabla 1. Indicadores del crecimiento y rendimiento en diferentes momentos de riego.

\begin{tabular}{|c|c|c|c|c|c|}
\hline Tratamientos & $\begin{array}{l}\text { Largo de } \\
\text { la hoja } \\
\text { (cm) }\end{array}$ & $\begin{array}{l}\text { Ancho } \\
\text { de la } \\
\text { hoja } \\
(\mathrm{cm})\end{array}$ & $\begin{array}{l}\text { Grosor } \\
\text { de la } \\
\text { hoja } \\
(\mathrm{cm})\end{array}$ & $\begin{array}{l}\text { Peso de } \\
\text { la hoja } \\
\text { (g) }\end{array}$ & $\begin{array}{c}\text { Peso del gel } \\
\text { (g) }\end{array}$ \\
\hline & \multicolumn{5}{|c|}{ Medias } \\
\hline $\begin{array}{l}\text { Control (sin } \\
\text { riego) }\end{array}$ & $44,42 d$ & $5,98 \mathrm{c}$ & $1,90 \mathrm{~d}$ & $230,87 d$ & $105,81 d$ \\
\hline Riego 8 am & $54,19 a b c$ & $7,29 a b$ & $2,46 b$ & $409,25 b$ & $257,00 \mathrm{~b}$ \\
\hline Riego 3 pm & $49,76 \mathrm{~cd}$ & $6,41 \mathrm{bc}$ & $2,39 \mathrm{c}$ & $365,62 c$ & $192,67 c$ \\
\hline Riego 8 pm & $52,56 \mathrm{bc}$ & $8,33 \mathrm{a}$ & $2,79 a$ & $514,62 \mathrm{a}$ & $326,49 \mathrm{a}$ \\
\hline EE $\bar{X}$ & 0,46 & 0,23 & 0,06 & 14,15 & 11,00 \\
\hline
\end{tabular}


Con relación al ancho de la hoja los mayores valores se obtuvieron en los tratamientos de riego aplicados a las 8.00 pm y 8.00 am con diferencias significativas con el control. La realización del riego a las 3.00 pm no tuvo diferencias significativa con el control.

El grosor y peso de la hoja, así como el peso del gel fue mayor en el tratamiento nocturno, con diferencias significativas con el resto de los tratamientos y un rendimiento estimado en hoja y gel de 61,752 Mg.ha ${ }^{-1}$ corte $^{-1}$ año y 39,180 Mg.ha ${ }^{-1} \cdot$ corte $^{-1}$ año respectivamente, seguido por el riego a las 8.00 am que manifestó diferencias significativas con el riego a las $3.00 \mathrm{pm}$ y el control, con un rendimiento de 49,104 Mg.ha ${ }^{-1} \cdot$ corte $^{-1}$ año y 30,840 Mg.ha ${ }^{-1} \cdot$ corte $^{-1}$ año. En estos indicadores los tratamientos con riego arrojaron diferencias significativas con el control, que alcanzó un rendimiento de 27,696 y 12,698 Mg.ha ${ }^{-1}$.corte ${ }^{-1}$ año de hoja y gel respectivamente.

Estos resultados demuestran que el cultivo de la sábila responde a los tratamientos con riego, donde se incrementa su crecimiento, desarrollo y la producción de gel, siendo más favorecidos con el riego nocturno.

En este sentido Rodríguez et al. (2007), señala que aunque la planta de sábila por su metabolismo tolera déficits de humedad, su fisiología es alterada por las condiciones de cultivo, que la disponibilidad de agua es esencial para alcanzar mayor tasa de crecimiento y número de hojas e hijuelos.

Por otra parte los rendimientos obtenidos en el municipio Colina con dos cortes al año en dos épocas distintas (Iluvia y sequia) fue de 46,396 Mg*ha-1*año-1 cercano al promedio de los valores máximos y mínimos de gel de 55 a $45 \mathrm{Mg}^{*}$ ha-1*año-1 (Molero, et al., 2013). 


\section{Conclusiones}

La aplicación de riego en el cultivo de la sábila favorece el crecimiento y desarrollo de la planta.

Con el riego nocturno (8.00 pm), se alcanzan los mejores resultados de los indicadores del crecimiento de la planta, así como de peso de la hoja $(514,62 \mathrm{~g})$ y de gel $(326,49 \mathrm{~g})$ con un rendimiento estimado en hoja y gel de $61,752 \mathrm{Mg}$ ha $^{-1}$ corte $^{-1}$ año y 39,180 Mg.ha ${ }^{-1} \cdot$ corte $^{-1}$ año respectivamente.

\section{REFERENCIAS CONSULTADAS}

1. Añez, B. y J. Vásquez, (2005). Efecto de la densidad de población sobre el crecimiento y rendimiento de la zábila (Aloe barbadensis M.), Rev. Fac. Agron. Vol. 22 No 1, p. 10-15, Caracas, Venezuela.

2. Bisceglia, M. (1999). Comercialización de zábila. Jornadas zábila en estado Falcón, acciones para su desarrollo. Instituto Universitario de Tecnología Alonso Gamero. Trabajo de Ascenso. Venezuela. 166 p.

3. Bozzi, A., Perrin, C., Austin, S., y Arce, F. (2007). Quality and autenticity of commercial Aloe vera gel powders. Food Chemistry No 10, p. 22-30.

4. Castañeda, L. (2001). Plan estratégico para el desarrollo sustentable de la sábila. Rubro prioritario del estado Falcón. [Disponible]: http://www.municipiourdaneta.com/sabilafalcon.php. [Consulta: 2014 febrero, 23].

5. Fábrica para procesamiento de sábila de Venezuela (SABILVEN S.A), (2014). Picado y Lavado de Hoja de Sábila para su Posterior Procesamiento, Gel de Áloe Vera. Falcón Venezuela.

6. Figueredo, C. y Morales, J. (2010). Plan integral para la comercialización de Aloe vera en Colombia. Trabajo de grado. Administración de Negocios Internacionales. Administración de Empresas. Facultad de Administración. Universidad del Rosario. $111 \mathrm{p}$.

7. Geydan, T. y Melgarejo, L. (2005). Metabolismo ácido de las crasuláceas. Acta Biológica Colombiana, Vol.10 No 2, p. 3-14. 
8. González Caneloríguez, M., Puertas Arias, A., Jiménez Arteaga, M., Danger Hechavarría, L., \& López Álvarez, S. (2018). USO DE Trichoderma harzianum Rifaii PARA EL CONTROL BIOLÓGICO DE Sclerotium rolfsii Sacc. EN EL CULTIVO DE ZÁBILA (Aloe vera L.). Revista Arbitrada Interdisciplinaria Koinonía, 2(3), 213-225. Recuperado de http://fundacionkoinonia.com.ve/ojs/index.php/revistakoinonia/article/view/60

9. López, R., Murcia, C., López, P., y Valencia, J. (2010). Estandarización del protocolo de desinfección de disco de hoja en la inducción de callogénesis de cordiaalliodora (ruiz\&pav.) okén (lamiales: boraginaceae) en condiciones in vitro. [Disponible]: http:// blade1. uniquindio. edu.co/ uniquindio / revista investigaciones. [Consultado:2014,enero 17].

10. MINISTERIO DE AGRICULTURA Y CRIA (MAC). (1975). Inventario Nacional de Tierras, Regiones: Costa nor-occidental, Centro-occidental y Central. Comisión del Plan Nacional de Aprovechamiento de los Recursos Hidráulicos (COPLANARH). Centro Nacional de Investigaciones Agropecuaria (CENIAP). Caracas, Venezuela. II 43 p..

11.MINISTERIO DE AMBIENTE Y DE LOS RECURSOS NATURALES (MARN) (2014). Datos mensuales de precipitación (Pp), evaporación (Ev) y humedad relativa $(\mathrm{Hr})$ del municipio Colina. Dirección de Hidrología, Meteorología y Oceanografía. Coro, Estado Falcón Venezuela.

12. Molero, T., Viloria, M., Patiño, D., y Ocando, M. (2013). Producción de gel y acíbar en plantaciones de sábila (Aloe barbadensis Mill) en el occidente de Venezuela. Bioagro 25 (1): 71-76.

13.Monasterio, P., Pierre, F., Barreto, T., Alejos, G., Maturet, W., y Tablante, J. (2008). El pluviómetro artesanal: una manera práctica de medir la precipitación. INIA Divulga, (11): 45-49.

14.Piña, H. (2005). Perfil preliminar del mercado de zabila (Aloe barbadensis Miller.) en el estado falcón, Venezuela. Bioagro, Vol 7 No 2, p.15-22.

15.Piña, H, Lugo, Z. Azócar, R. y Romero, C. (2005). Tipología de la producción primaria de zábila (Aloe vera L.) en el estado Falcón, Venezuela. Revista problemas del desarrollo, Vol. 41, No 16, p.187-208.

16. Pulido, F. (2014). Cultivo de sabila (Aloe vera L). Boletín informativo "inédito". Universidad Esperimental Francisco de Miranda. Falcon, Venezuela, $63 \mathrm{p}$. 
KOINONIA. Revista Arbitrada Interdisciplinaria de Ciencias de la Educación, Turismo, Ciencias Sociales y Económica, Ciencias del Agro y Mar y Ciencias Exactas y aplicadas. Año IV. Vol IV. Nº . Enero - Junio 2019. Hecho el depósito de Ley: FA2016000010 ISSN: $2542-3088$

FUNDACIÓN KOINONIA (F.K). Santa Ana de Coro. Venezuela.

Víctor Alfonso Blanco Bello; Juan Pedro Machado Castillo; Norge Tornes Olivera

17.Rodríguez, R., Jaso, D., Gil, J., Angulo, J., y Lira, R. (2007). Crecimiento, resistencia estomática y transpiración de Aloe vera con diferentes potenciales hídricos del suelo. Ind. Crops Prod. Vol. 25 No. 2, p.123-128.

18. Sistema de Inteligencia de Comercio Exterior (1997). Reporte estadístico. Exportaciones no tradicionales, código 1302190090.Venezuela. Informe técnico. $24 \mathrm{p.}$

C2019 por los autores. Este artículo es de acceso abierto y distribuido según los términos y c ondiciones de la licencia Creative Commons Attribution

(http://creativecommons.org/licenses/by/4.0/). 